\title{
Análisis exploratorio de la imagen turística de la ciudad de Granada por parte de una muestra de estudiantes de lengua española
}

\author{
María Eulalia MARTÍNEZ GARCÍA \\ Máster en Dirección Turística Internacional \\ Universidad Rey Juan Carlos \\ me.martinezga@alumnos.urjc.es \\ Juan José FERNÁNDEZ MUÑOZ \\ Departamento de Psicología \\ Universidad Rey Juan Carlos de Madrid \\ Campus de Alcorcón; Madrid, 28922 \\ juanjose.fernandez@urjc.es \\ José Manuel CASTAÑo BLANCO \\ Departamento de Psicología \\ Universidad Rey Juan Carlos de Madrid \\ Campus de Fuenlabrada; Madrid, 28943 \\ josemanuel.castano@urjc.es
}

Recibido: 07-03-2013

Aceptado: 18-04-2013

\begin{abstract}
RESUMEN
La imagen de un destino turístico influye en gran medida sobre el proceso de decisión de compra por parte del turista. Entender el proceso de formación de dicha imagen y comprender la importancia de las dimensiones que la integran ha sido uno de los temas más investigados en materia turística. El presente estudio trata de analizar la imagen percibida de la ciudad de Granada por un grupo de turistas cuya motivación principal es aprender la lengua española. Se han examinado las respuestas que dieron a un cuestionario una muestra compuesta por 209 estudiantes, con una media de edad de 21.71 años, del Centro de Lenguas Modernas (de ahora en adelante, CLM) dependiente de la Universidad de Granada. Los datos recopilados se han analizado mediante Anova y método Clúster.
\end{abstract}


Palabras claves: imagen-destino, formación de la imagen, destino turístico, Granada, lengua española, análisis Clúster.

\title{
Exploring the touristic image of Granada by a sample of Spanish language students
}

\begin{abstract}
The image of a destination has a strong influence on an individual's buying decision making process. Understanding the formation of the tourist image and the importance of its dimensions has been one of the most investigated topics in tourism investigations. The current study deals with analyzing the image perceived of Granada according to a certain tourists whose main motivation consists of learning Spanish language. A questionnaire was conducted on a sample of 209 students with an average age of 21.71. Students belong to Centro de Lenguas Modernas which depends on Universidad de Granada. Answers have been analyzed by using different statistical methods: anova and clúster method.
\end{abstract}

Keywords: destination image, formation of destination image, tourism destination, Granada, Spanish language, clúster.

Sumario: 1. Introducción 2. Marco conceptual 3. Objetivos 4. Metodología 5. Resultados 6. Discusión.

\section{INTRODUCCIÓN}

\section{Importancia del estudio de la imagen de un destino turístico}

Hoy en día el turismo es uno de los sectores que más contribuye al desarrollo económico de un país y de las regiones que lo componen (Organización Mundial del Turismo, de ahora en adelante OMT, 1998). En el caso de España, este sector constituye uno de los motores más importantes de la economía por los ingresos que genera esta actividad y porque se conforma como una importante fuente de empleo (Rial, García y Varela, 2008).

De forma paralela al favorable papel que desempeña el sector turístico en la economía, cada vez es más notable el incremento de competencia entre productos y destinos turísticos. Este hecho junto a los cambios y expectativas de los turistas, la mayor exigencia por parte de los clientes y el aumento en la promoción turística por parte de los destinos conlleva que éstos sean concebidos como marcas que deben ser gestionadas con una visión estratégica (Beerli y Martín, 2002; Moreno, Beerli y De León Ledesma, 2012).

Además como consecuencia de la globalización y de la apertura de las fronteras nacionales al intercambio, los turistas se encuentran entre un mayor número de alternativas a la hora de elegir un destino. Por tanto, resulta fundamental que los destinos constituyan una imagen positiva, diferenciada y reconocible del lugar en 
sus mercados objetivos para lograr una ventaja competitiva sobre otros destinos alternativos (Baloglu y McCleary, 1999b; Echtner y Ritchie, 2003; Gartner, 1993). Esta imagen será decisiva a la hora de atraer turistas hacia un lugar (Ahmed, 1991).

\section{Estudio de la lengua española como recurso cultural y turístico}

Hoy en día, el español se constituye como lengua oficial en 20 países, es la segunda lengua de comunicación internacional y la cuarta lengua más hablada del mundo por una cifra aproximada de 400 millones de personas. En los últimos años se ha experimentado un creciente interés por el estudio de la lengua y cultura española. Esta actividad ha llegado a consolidarse como un subsegmento del turismo cultural, denominada turismo idiomático, cuya principal motivación es el aprendizaje de la lengua y adquirir un mayor grado de conocimiento de la cultura, costumbres y sociedad del destino. Esta actividad suele combinarse con la práctica de actividades turísticas complementarias, se realiza en un país diferente al habitual del turista y por un período de tiempo consecutivo e inferior a un año (Hernández y Campón, 2010).

Tabla 1. Aproximación al concepto imagen-destino.

\begin{tabular}{|c|c|}
\hline AUTOR & DEFINICIÓN \\
\hline Hunt (1975) & Las percepciones que tienen los visitantes potenciales sobre un área \\
\hline Lawson y Baud-Bovy (1977) & $\begin{array}{l}\text { La expresión de todas las creencias, impresiones, prejuicios y / o pensamientos } \\
\text { emocionales que los individuos tienen de un lugar específico }\end{array}$ \\
\hline Crompton (1979) & $\begin{array}{l}\text { La suma de las creencias, ideas e impresiones que una persona tiene sobre un destino } \\
\text { turístico }\end{array}$ \\
\hline Assael (1984) & $\begin{array}{l}\text { La percepción total que tienen los individuos sobre un lugar, la cual se forma a } \\
\text { través de varias fuentes de información }\end{array}$ \\
\hline Phelps (1986) & Percepciones o impresiones sobre un lugar \\
\hline Gartner (1986) & $\begin{array}{l}\text { La percepción de los turistas y vendedores sobre los atributos de las actividades o } \\
\text { atractivos disponibles en un destino }\end{array}$ \\
\hline Fridgen (1987) & Representación mental de un lugar que no está físicamente delante del observador \\
\hline Gartner y Hunt (1987) & Impresiones que una persona tiene de un estado en el que no reside \\
\hline
\end{tabular}




\begin{tabular}{|c|c|}
\hline Moutinho (1987) & $\begin{array}{l}\text { Actitud del turista hacia un conjunto de atributos del destino basada en sus } \\
\text { conocimientos y sentimientos }\end{array}$ \\
\hline Gartner (1989) & Una combinación compleja de varios productos y atributos asociados \\
\hline Reilly (1990) & $\begin{array}{l}\text { No es sólo un conjunto de rasgos o cualidades individuales, sino también una } \\
\text { impresión general que sobre una entidad se forma en la mente de los individuos }\end{array}$ \\
\hline Um y Crompton (1990) & $\begin{array}{l}\text { Gestalt o constructo holístico que se deriva de las actitudes hacia los atributos } \\
\text { turísticos que se perciben del destino }\end{array}$ \\
\hline Chon (1990) & $\begin{array}{l}\text { Resultado de la interacción de las creencias, ideas, sentimientos, expectativas e } \\
\text { impresiones de una persona sobre un destino }\end{array}$ \\
\hline Echtner y Richtie (1991) & $\begin{array}{l}\text { Las percepciones que tienen los individuos sobre los atributos, tanto funcionales } \\
\text { como psicológicos del destino, y la impresión holística que se forma del destino }\end{array}$ \\
\hline Fayeke y Crompton (1991) & $\begin{array}{l}\text { Constructo mental desarrollado por un visitante potencial sobre la base de unas } \\
\text { pocas impresiones seleccionadas entre un flujo total de impresiones }\end{array}$ \\
\hline Stern y Krakover (1993) & Conjunto de sentimientos e impresiones individuales sobre un lugar \\
\hline Milman y Pizam (1995) & $\begin{array}{l}\text { Impresión mental o visual de un lugar o producto que experimenta el público en } \\
\text { general }\end{array}$ \\
\hline $\begin{array}{l}\text { MacKay y Fesenmaier } \\
\text { (1997) }\end{array}$ & $\begin{array}{l}\text { Impresión global compuesta por varias atracciones y atributos del destino que se } \\
\text { muestran entrelazados }\end{array}$ \\
\hline OMT (1998) & Son las ideas o conceptos que se tienen de un destino a nivel individual o colectivo \\
\hline Pritchard (1998) & Una impresión visual o mental de un lugar específico \\
\hline $\begin{array}{l}\text { Baloglu y MacCleary } \\
(1999 a)\end{array}$ & $\begin{array}{l}\text { Representación mental de las creencias, los sentimientos y la impresión global del } \\
\text { individuo sobre un destino }\end{array}$ \\
\hline $\begin{array}{l}\text { Murphy, Pritchard y Smith } \\
(2000)\end{array}$ & El conjunto de asociaciones y piezas de información relacionadas con un destino \\
\hline
\end{tabular}




\begin{tabular}{|l|l|}
\hline $\begin{array}{l}\text { Bigné, Sánchez y Sánchez } \\
(2001)\end{array}$ & Interpretación subjetiva de la realidad hecha por un turista \\
\hline Kim y Richardson (2003) & $\begin{array}{l}\text { La totalidad de impresiones, creencias, ideas, expectativas y sentimientos de un lugar } \\
\text { acumulados con el paso del tiempo }\end{array}$ \\
\hline $\begin{array}{l}\text { Camprubí, Guia y Comas } \\
(2009)\end{array}$ & $\begin{array}{l}\text { La imagen turística es una construcción social resultante de la interacción de los } \\
\text { distintos agentes que intervienen en el destino turístico (administraciones públicas, } \\
\text { instituciones locales, empresas turísticas, etc.) }\end{array}$ \\
\hline $\begin{array}{l}\text { Del Barrio, Luque y } \\
\text { Rodríguez (2009) }\end{array}$ & $\begin{array}{l}\text { Conjunto de adjetivos de una ciudad relacionados con un estímulo dado (físico o } \\
\text { social), que conlleva que los públicos objetivos formen unas asociaciones positivas o } \\
\text { negativas sobre la ciudad. Estas asociaciones, de naturaleza cognitiva o afectiva, } \\
\text { componen un cuerpo de conocimiento denominado "creencias o estereotipos". }\end{array}$ \\
\hline
\end{tabular}

Fuente: Elaboración propia a partir de Echtner y Richtie (2003); Gallarza, Gil y Calderón (2002) y San Martín (2005, 2008).

Por todo ello se puede afirmar que el turismo idiomático resulta de gran interés para el turismo español. En primer lugar, por su contribución al desarrollo económico del destino al ser muy prolongada la estancia media del turista, elevado el gasto medio del visitante y notable el consumo de productos complementarios. En segundo lugar, por ser un producto que se oferta en ciudades de gran riqueza y patrimonio histórico-cultural (Salamanca, Alcalá de Henares, Sevilla, Granada, etc.) y que permite afianzar la imagen turística del destino. Y por último, por constituirse como un producto que contribuye a la ruptura de la estacionalidad y que supone una fidelización del turista con el destino, ya que es común que el estudiante vuelva a visitarlo acompañado de familiares y amigos.

\section{MARCO CONCEPTUAL \\ Conceptualización de la imagen de un destino turístico}

Desde la década de los 70 son numerosos los estudios que se han llevado a cabo con el propósito de conceptualizar y delimitar el término de imagen turística así como de explicar el proceso de formación de tal imagen. En la tabla 1 se recogen las definiciones aportadas por algunos de los investigadores que a través de sus estudios han intentado aproximarse a una definición de este concepto.

La revisión bibliográfica pone de manifiesto la falta de consenso en una definición precisa de este término. Diversos autores coinciden en afirmar que este concepto ha sigo vagamente definido (Baloglu y McCleary, 1999a; Beerli y Martín, 2004a; Fakeye y Crompton, 1991; Gallarza, Gil y Calderón, 2002) ya que éste es descrito simplemente como "impresiones" o "percepciones" que una persona percibe de un destino (Echtner y Ritchie, 2003). 
Algunos autores se centran en la naturaleza holística de la imagen, considerándola como una impresión global que es mayor que la suma de las partes individuales (Fakeye y Crompton, 1991). En cambio, otros autores como Crompton (1979) coinciden en afirmar que la imagen de un destino se compone de la percepción de sus atributos individuales.

En el estudio de Gallarza et al. (2002) se lleva a cabo una exhaustiva revisión bibliográfica sobre este concepto en trabajos previos. Como resultado estos autores llegan a la conclusión de que el término imagen-destino es un concepto de naturaleza compleja (carece de un único significado ya que permite más de una interpretación), múltiple en procesos y elementos, relativista (de carácter subjetivo y comparativo) y dinámico en el tiempo y en el espacio.

De lo expuesto anteriormente destaca el carácter subjetivo de dicho concepto (Bigné, Sánchez y Sánchez, 2001; San Martín y Rodríguez, 2008). Igualmente, Traverso (2007) enfatiza la subjetividad y carácter dinámico de tal concepto "la imagen de un destino no es homogénea para todos los públicos, ni permanente en el tiempo. Por esto debemos de hablar de imagen de un determinado destino en la mente de un determinado colectivo y para un momento temporal concreto" (p.41). En este sentido, Del Barrio, Luque y Rodríguez (2009) resaltan que la estructura dinámica y sensible de la imagen puede explicarse por los cambios que afectan al entorno en el que el estímulo se inserta, o por los cambios que experimenta la persona que evalúa dicho estímulo.

Una de las definiciones más completas sobre el término imagen-destino es la que expresan Baloglu y McCleary (1999a) considerando la imagen como la representación mental de las creencias, los sentimientos y la impresión global del individuo sobre un destino. Ésta integra tanto las impresiones del turista como las evaluaciones cognitivas o perceptuales (aquellas creencias y conocimientos que tiene un individuo sobre las características y los atributos de un lugar) y las evaluaciones afectivas o evaluativas (las respuestas emocionales que los individuos expresan de un determinado destino). El conjunto de ambas evaluaciones configura la imagen global o compuesta la cual puede ser similar o diferir de las evaluaciones cognitivas o afectivas.

En el trabajo de Pike (2002) se lleva a cabo una revisión bibliográfica de 142 documentos de investigación sobre imagen y destino que fueron publicados entre 1973 y 2000. El autor concibe su trabajo como una guía que recoge las principales características sobre la investigación en este ámbito de estudio. De todos los documentos revisados, Pike menciona que en sólo 6 de ellos se pone de manifiesto un interés explicito por el análisis del componente afectivo.

\section{Modelos de formación de imágenes}

En la revisión bibliográfica sobre imagen-destino se pone de manifiesto que son numerosos los investigadores que mediante diferentes modelos teóricos han tratado 
de esclarecer el proceso de formación de la imagen turística, así como los factores que resultan más influyentes en la elección de un destino turístico.

Reynolds (1965, c.p. Echtner y Richtie, 2003) considera el proceso de formación de la imagen como el desarrollo de un constructo mental basado sobre algunas impresiones seleccionadas de diversas fuentes de información.

Posteriormente, Gunn (1972) propone un modelo compuesto de siete fases en el que las fuentes de información adquieren especial relevancia en el proceso de formación de la imagen. Las fases que configuran dicho modelo son: (1) acumulación de la imágenes mentales sobre experiencia vacacional, conformándose una imagen orgánica; (2) modificación de dichas imágenes con información adicional, constituyéndose una imagen inducida; (3) decisión de hacer el viaje de vacaciones; (4) viaje al destino elegido; (5) inmersión en el destino y participación de actividades; (6) vuelta a casa, y (7) modificación de imágenes tras la experiencia.

El modelo de Baloglu y McCleary (1999a) no solo incluye la experiencia viajera y las fuentes de información como factores claves para explicar el proceso de formación de la imagen, sino que también incorpora las variables psicosociales del individuo. Estos autores distinguen entre factores personales y factores estímulo. Los personales o internos hacen referencia a variables psicosociales (valores, motivación y personalidad) y sociales (edad, educación y estado civil) del individuo. Por otro lado, los factores estímulo son externos al individuo y comprenden las fuentes de información y la experiencia previa. Respecto a estas dos últimas variables, Echtner y Richtie (2003) difieren del modelo propuesto, defendiendo que se puede constituir una imagen de un destino sin haberlo visitado previamente y sin haber consultado fuentes de información relacionadas con el mismo. 
Figura 1. Modelo de proceso de formación de la imagen de Gunn

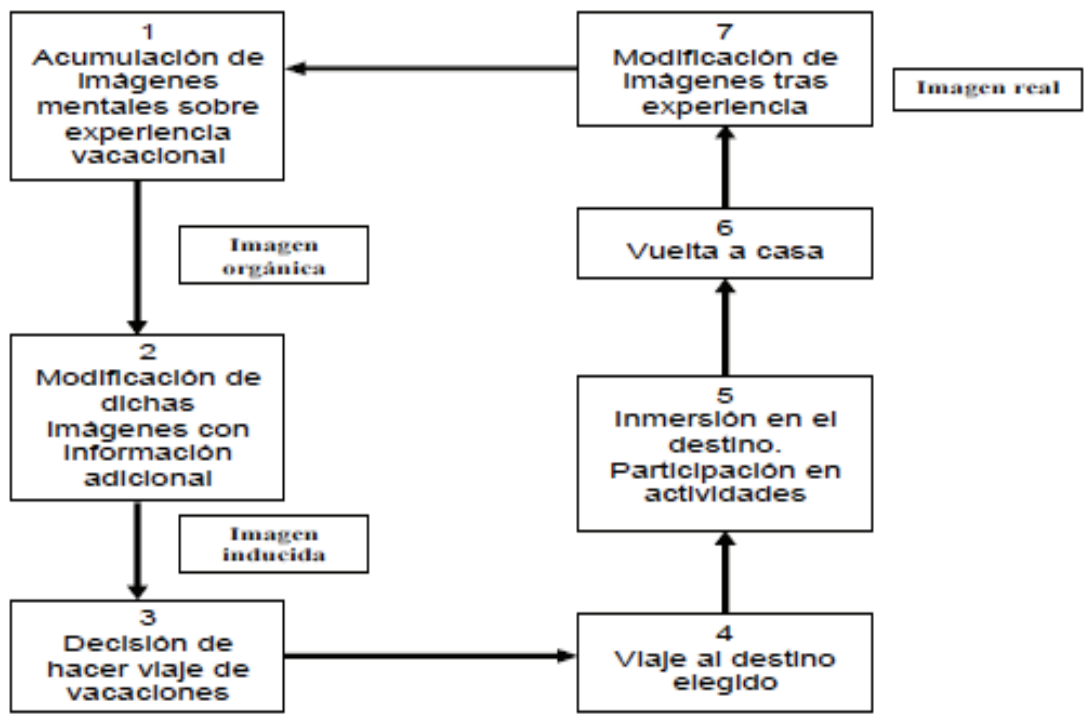

Fuente: Castaño, Moreno y Crego (2006)

Basándose en el modelo de Baloglu y McCleary (1999a) cabe reseñar que entre los factores personales más destacados en la literatura sobre imagen turística que afectan la percepción del cliente se encuentran las motivaciones, los valores culturales y las características sociodemográficas del individuo.

Respecto a la motivaciones, estas se entienden como necesidades psicológicas del individuo que provocan un desequilibrio que puede subsanarse mediante la experiencia turística (Kim y Lee, 2002). La mayoría de los estudios que indagan sobre la influencia de las motivaciones en la percepción de la imagen de un destino lo relacionan con el componente afectivo de la imagen (Gartner, 1993; Baloglu, 1997; Baloglu y McCleary, 1999a; Beerli y Martín, 2004c), esto se explica porque las motivaciones están vinculadas con valores emocionales e internos de los individuos (Yoon y Uysal, 2005). 
Figura 2. Proceso de formación de la imagen - destino de Baloglu y McCleary

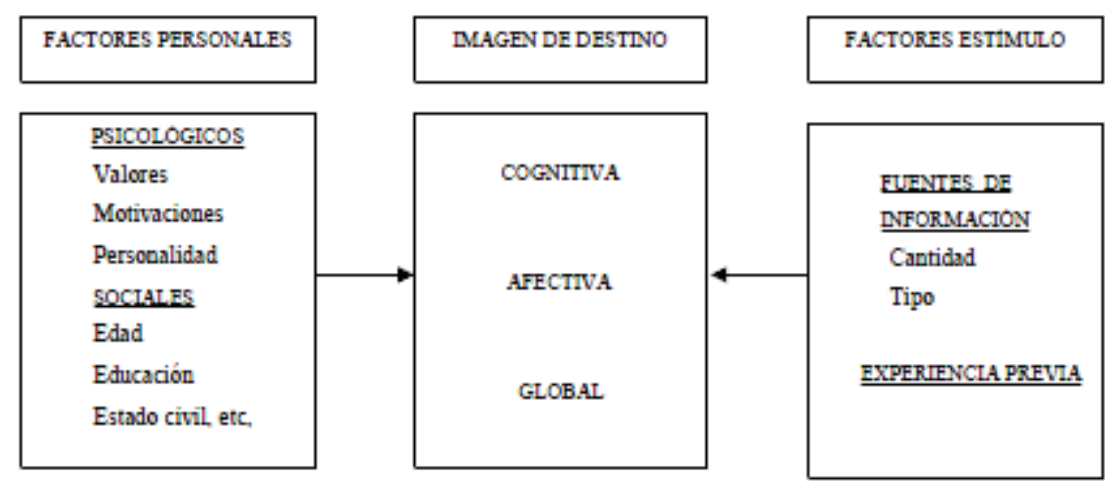

Fuente: Elaboración propia a partir de Baloglu y McCleary (1999a).

En relación con los valores culturales del individuo cabe comentar que en la investigación turística estos valores se abordan desde la perspectiva del origen geográfico del turista (San Martín y Rodríguez, 2010a). De este modo, se constata que la imagen cognitiva de un destino se ve influenciada por el país de origen del viajero (Chen y Kerstetter, 1999). Asimismo, Lee y Lee (2009) indican que la imagen que los turistas se forman en la mente sobre un destino y su comportamiento en ese lugar puede ser reflejo de los valores culturales de la sociedad de la que proceden.

En este sentido, Moreno et al. (2012) recalcan la necesidad de llevar a cabo una mayor investigación sobre las diferencias en la percepción de la imagen de un lugar en función de la nacionalidad de los turistas. Esto se explica por la dificultad de realizar estudios interculturales debido a la problemática del idioma, la interpretación de las escalas y la complejidad para obtener submuestras procedentes de diferentes nacionalidades que sean comparables.

Por su parte, Mackay y Fesenmaier (1997) consideran que cuánto menor es la distancia cultural, al turista le resulta más familiar el destino y lo percibe de forma más favorable. Por el contrario, un exceso de familiaridad puede provocar que el destino resulte menos atractivo para el visitante. Wang y Hsu (2010) vinculan la familiaridad con las visitas previas al destino, comprendiendo la experiencia directa con un lugar como un factor que puede llegar a modificar la imagen del visitante sobre este último.

Por último, el modelo de Baloglu y McCleary (1999a) expone que las características sociodemográficas del individuo es otro de los factores que influyen en la percepción de la imagen. En la literatura académica se constata un relevante interés en analizar si variables tales como la edad, el género, el nivel de educación, 
la ocupación, el nivel de ingresos o el estado civil influyen en el proceso de formación de la imagen.

\section{OBJETIVOS}

Se plantea como objetivo principal del estudio analizar la naturaleza de la imagen de la ciudad de Granada por parte de un grupo de estudiantes de español mediante la identificación de las distintas dimensiones que componen la imagen percibida de este destino.

Siguiendo esta línea como base para alcanzar este objetivo general, se plantean los siguientes objetivos específicos:

(I) Análisis exploratorio de la imagen de Granada determinando si existen diferencias significativas en función de la nacionalidad de los estudiante

(II) Establecer posibles agrupaciones o clúster de estudiantes con perfiles sociales similares y que se caractericen por compartir una imagen homogénea de la imagen turística de esta ciudad.

(III) Identificación de elementos del componente único y holístico de la imagen de Granada.

\section{METODOLOGÍA}

Para dar respuesta a los objetivos anteriores se plantearon dos fases de trabajo: revisión bibliográfica de trabajos previos sobre imagen-destino y una fase cuantitativa mediante el diseño de un cuestionario.

\section{Muestra}

El número de alumnos matriculados en cursos de español en el CLM durante el mes de abril de 2012 asciende a 1193 individuos. La muestra encuestada se compone de 209 estudiantes de los cuales el $22.50 \%$ son hombres, mientras que el $77.50 \%$ son mujeres, y cuyas edades se encuentran en un rango de edad comprendido entre 18 y 44 años $(\mathrm{M}=21.71 \mathrm{DT}=3,11)$. En relación con la procedencia de los individuos los americanos representan el $61.4 \%$ de la muestra, los europeos el $31.7 \%$ y los asiáticos el $6.9 \%$. De los estudiantes encuestados tan solo el $27.8 \%$ pertenecen al programa "Erasmus" de intercambio entre Universidades Europeas.

\section{Procedimiento}

El trabajo de campo se lleva a cabo durante el mes de Abril de 2012, en concreto, durante el miércoles 25 y jueves 26 en ambas sedes del CLM, tanto en el antiguo Palacio de Santa Cruz del siglo XVI, como en el palacete de la Huerta de los 
Ángeles. En la tabla 2 se recoge una ficha técnica que resume el proceso metodológico empleado.

Tabla 2. Ficha técnica del proceso metodológico

\begin{tabular}{|l|l|}
\hline Universo & $\begin{array}{l}\text { Estudiantes matriculados en algún curso de español en } \\
\text { el CLM de Granada durante el mes de abril 2012 (1193) }\end{array}$ \\
\hline Ámbito geográfico & Ciudad de Granada \\
\hline Recogida de información & Cuestionario personal. Encuesta \\
\hline Tamaño de la muestra & 209 \\
\hline Error muestral & $+/-6.29 \%$ \\
\hline Nivel de significación & $95.5 \% \quad \mathrm{Z}=2 \quad(\mathrm{p}=\mathrm{q}=0,5)$ \\
\hline Fecha del trabajo de campo & Abril 2012 \\
\hline
\end{tabular}

Fuente: Elaboración propia

\section{Instrumento}

Previamente al diseño del cuestionario se realiza una revisión de material promocional sobre la ciudad de Granada para identificar los atributos más relevantes de este destino. Igualmente, se revisan escalas empleadas en trabajos previos (Baloglu y McCleary, 1999b; Beerli y Martín, 2004a, 2004c; Bigné y Sánchez, 2001; Castaño et al., 2006; Moreno, Beerli y Martin, 2004; San Martín, Rodríguez y Vázquez, 2006) que permiten identificar atributos de la imagen susceptibles de ser incorporados en el cuestionario, adecuándose a las características específicas del destino objeto de estudio y al perfil de los turistas que acuden al mismo.

En los cuestionarios se incluye una breve explicación del estudio y se indica a los participantes que sus respuestas se tratarán de manera anónima y con la máxima confidencialidad, y serán evaluadas de manera conjunta y no individualmente.

El cuestionario se estructura en cuatro bloques:

1) Variables sociodemográficas, tales como el género, edad, país de procedencia y formación del individuo. Se incluye una pregunta referente a si el encuestado pertenece o no al programa "Erasmus" de Intercambio entre Universidades Europeas, así como variables turísticas y culturales relacionadas con el estudio de español en Granada (fuentes a través de las que se ha informado el encuestado, si es su primera visita a esta ciudad, duración media, motivo por el que estudia español, nivel de español, motivo por el que ha 
elegido la ciudad de Granada para estudiar español y el tipo de alojamiento escogido).

2) Percepción de la imagen de Granada. Este bloque se subdivide en tres subgrupos (componente cognitivo, componente afectivo e imagen global) compuesto de 33 items. 1) Componente cognitivo. Consta de 28 afirmaciones que hacen referencia a aspectos tales como "facilidad de acceso desde otras regiones o países", "oferta de alojamiento de calidad", "amplia oferta de restauración", "facilidades para ir de compras", "buen nivel de infraestructuras", "riqueza histórica y patrimonial", "dispone de numerosas atracciones culturales para visitar", "actividades culturales de interés", "costumbres y tradiciones dignas de conocer", "el flamenco es uno de los rasgos más característicos de la ciudad", "los espectáculos taurinos son uno de los rasgos más característicos de la ciudad", "ciudad idónea para aprender español", "destino con riqueza y belleza paisajística", "clima agradable", "ofrece oportunidades para la aventura y el deporte", " gastronomía es rica, variada y de calidad", "la cultura del tapeo es uno de los rasgos más característicos de la ciudad", "habitantes amables y hospitalarios", "vida nocturna atractiva", "destino sin contaminación ambiental, limpio y cuidado", "destino con alta estabilidad política y social", "destino masificado", "lugar seguro para visitar", "lugar con buena atención al turista y que cuida la calidad del servicio", "buena relación calidad/precio", "oficinas de información turística son fácilmente localizables por los turistas", "señalización de los monumentos es buena" y "antes de viajar a Granada disponía de amplia información sobre la ciudad". Se emplea una escala tipo Likert con valores comprendidos entre 1 y 5 , donde 1 es totalmente en desacuerdo y 5 totalmente de acuerdo. 2) Componente afectivo. Incluye la valoración de los atributos afectivos a través de una escala de diferencial semántico de 5 posiciones (aburrido - divertido; estresante - relajante, deprimente - excitante, desagradable - agradable). 3) Imagen global. Mide a través de una pregunta la imagen global de Granada mediante una escala Likert de 5 valores, donde 1 es muy insatisfactorio y 5 muy satisfactorio.

3) Variables de comportamiento. Se compone de dos preguntas que miden la intención del encuestado de regresar a Granada en un futuro y la intención de que éste recomiende visitar la ciudad a sus amigos, familiares y conocidos.

4) Pregunta abierta. Consta de una pregunta abierta mediante la cual el entrevistado expone tres palabras que a su juicio describen la ciudad de Granada como destino turístico.

El cuestionario fue traducido al inglés para facilitar su comprensión por aquellos estudiantes con un nivel más básico de la lengua española. 


\section{Análisis}

Una vez finalizado el trabajo de campo y recogidos los cuestionarios, la información se analiza por medio del programa estadístico SPSS (Statistical Program for Social Sciences), versión 17.0 para Windows.

En primer lugar, para determinar si existen diferencias significativas en la imagen percibida de la ciudad de Granada en función de la nacionalidad del estudiante, se emplea el estadístico ANOVA. Se comprueba la significatividad a través del estadístico F de Fisher-Snedecor y el nivel crítico asociado, así como a través de los valores medios manifestados por los individuos de la muestra para cada factor de la imagen. En segundo lugar, para comprobar la posible agrupación de estudiantes en clúster se procede a un análisis de conglomerados en dos fases. Para determinar las variables categóricas significativas se emplea el test de ajuste estadístico chi-cuadrado, mientras que para las variables continuas se emplea la prueba t-student al 95\% del nivel de confianza. En tercer lugar, para analizar los resultados obtenidos en la pregunta abierta se utiliza un documento de Excel para realizar asociaciones de sinónimos y análisis de frecuencias.

\section{RESULTADOS}

\section{Anova}

En este epígrafe se trata de analizar la relación existente entre las distintas variables cognitivas y los tres bloques de nacionalidad de los encuestados (americanos, europeos y asiáticos), estableciendo si existen diferencias significativas entre dichas variables y nacionalidades a través del estadístico ANOVA. Se consideran las variables con valores $\mathrm{p}$ por debajo de .05 como estadísticamente significativas. 
Tabla 3. Anova variables cognitivas

\begin{tabular}{|c|c|c|c|c|c|c|}
\hline & Nacionalidad & $\mathrm{N}$ & M & DT & F & Sig \\
\hline \multirow{4}{*}{$\begin{array}{l}\text { Alojamiento de } \\
\text { calidad }\end{array}$} & Americanos & 124 & 4,15 & 902 & & \\
\hline & Europeos & 64 & 3,80 & .912 & & \\
\hline & Asiáticos & 14 & 3,29 & .825 & & \\
\hline & Total & 202 & 3,98 & .930 & $7,782^{*}$ & .001 \\
\hline \multirow{4}{*}{$\begin{array}{l}\text { Facilidades para ir de } \\
\text { compras }\end{array}$} & Americanos & 124 & 4,46 & .820 & & \\
\hline & Europeos & 64 & 4,22 & .766 & & \\
\hline & Asiáticos & 14 & 3,93 & .997 & & \\
\hline & Total & 202 & 4,35 & .828 & $3,809 *$ & .024 \\
\hline \multirow{4}{*}{$\begin{array}{l}\text { Costumbres y } \\
\text { tradiciones dignas de } \\
\text { conocer }\end{array}$} & Americanos & 124 & 4,41 & .733 & & \\
\hline & Europeos & 60 & 4,17 & .785 & & \\
\hline & Asiáticos & 14 & 3,86 & 1,099 & & \\
\hline & Total & 198 & 4,30 & .792 & $4,416^{*}$ & .013 \\
\hline \multirow{4}{*}{ Espectáculos taurinos } & Americanos & 123 & 2,74 & 1,108 & & \\
\hline & Europeos & 62 & 2,48 & 1,141 & & \\
\hline & Asiáticos & 14 & 3,50 & 1,019 & & \\
\hline & Total & 199 & 2,71 & 1,134 & $4,852^{*}$ & .009 \\
\hline \multirow{4}{*}{$\begin{array}{l}\text { Ciudad idónea para } \\
\text { aprender espanol }\end{array}$} & Americanos & 124 & 4,25 & .942 & & \\
\hline & Europeos & 64 & 4,08 & 1,145 & & \\
\hline & Asiáticos & 14 & 3.50 & 1,019 & & \\
\hline & Total & 202 & 4,14 & 1,029 & $3,626^{*}$ & .028 \\
\hline \multirow{4}{*}{$\begin{array}{l}\text { Oportunidades para la } \\
\text { aventura y el deporte }\end{array}$} & Americanos & 124 & 4,38 & .852 & & \\
\hline & Europeos & 63 & 3,92 & 1,036 & & \\
\hline & Asiáticos & 14 & 4,29 & .726 & & \\
\hline & Total & 201 & 4,23 & .926 & $5,373^{*}$ & .005 \\
\hline \multirow{4}{*}{$\begin{array}{l}\text { Estabilidad politica y } \\
\text { social }\end{array}$} & Americanos & 121 & 3,70 & .882 & & \\
\hline & Europeos & 63 & 3,16 & .971 & & \\
\hline & Asiáticos & 14 & 3,36 & 1,082 & & \\
\hline & Total & 198 & 3,51 & .955 & $7,348 *$ & .001 \\
\hline \multirow{4}{*}{$\begin{array}{l}\text { Lugar seguro para } \\
\text { visitar }\end{array}$} & Americanos & 124 & 4,45 & .691 & & \\
\hline & Europeos & 64 & 4,27 & .648 & & \\
\hline & Asiáticos & 14 & 4,00 & .679 & & \\
\hline & Total & 202 & 4,36 & .686 & $3,737^{*}$ & .026 \\
\hline
\end{tabular}

$$
* \mathrm{p}<.05
$$

Fuente: Elaboración propia

De este análisis se extrae que los americanos le conceden más importancia que los estudiantes de nacionalidad europea y asiática a variables tales como "alojamiento de calidad que ofrece la ciudad", $F(1,201)=7.782, p=.001$; "facilidades para ir de compras", $F(1,201)=3.809 p=.024$; "costumbres y tradiciones dignas de conocer", $F(1,197)=4.416, p=.013$; "ciudad idónea para aprender español", $F(1,201)=3.626, p=.028$; "ciudad que ofrece oportunidades para la aventura y el deporte", $F(1,200)=5.373, p=.005$; "destino con alta 
estabilidad política y social", $F(1,197)=7.384, p=.001$ y "lugar seguro para visitar", $F(1,201)=3.737, p=.026$. Por otra parte, los estudiantes de procedencia asiática perciben que los "espectáculos taurinos son unos de los rasgos más característicos de la ciudad", $F(1,198)=4.852, p=.009$, seguido de los estudiantes americanos, y por último, de los europeos que son los que le conceden menor importancia a esta variable.

\section{Clúster o conglomerado}

Para cumplir con el segundo objetivo se ha aplicado el método clúster mediante el cual todos los individuos han sido agrupados en dos clúster. Los sujetos de cada uno de los clúster se caracterizan por compartir unos rasgos comunes y homogéneos entre sí, pero diferenciados y heterogéneos respecto al otro clúster.

\section{Variables categóricas}

El primer clúster engloba al 47,9\% del total de los encuestados. Las nacionalidades más relevantes son la europea y asiática, siendo el $87,8 \%$ del total de estudiantes europeos y el $76,9 \%$ del total de estudiantes asiáticos. Respecto al nivel de español, este grupo se caracteriza por poseer un nivel básico-intermedio, agrupando al $82,1 \%$ del total de encuestados que indican poseer un nivel básico de español y al 50,6\% del total de encuestados que señalan que su nivel es intermedio. En relación con el tipo de alojamiento escogido, este conglomerado engloba al $81,5 \%$ del total de encuestados que eligen un piso de estudiantes durante su estancia en Granada para aprender español.

En cambio, el segundo clúster está representado por el 52,1\% del total de los entrevistados. La nacionalidad predominante es la americana, siendo el 73,8\% del total de estudiantes americanos entrevistados los que forman parte del mismo. Uno de los rasgos más característicos de este grupo es que integra al 72,4\% del total de encuestados que afirman poseer un nivel avanzado de español. Este conglomerado engloba también al $74,4 \%$ del total de encuestados que eligen la casa de una familia española como lugar de residencia.

\section{Variables continuas}

En este apartado se presentan los resultados obtenidos para cada uno de los clúster en relación con el conjunto de variables continuas que hacen referencia a la dimensión cognitiva de la imagen. Se toma como referencia el parámetro estadístico $t$-student al 95\% del nivel de confianza.

El conglomerado compuesto principalmente por estudiantes de nacionalidad asiática y europea valora de manera más negativa que para la media del conjunto de variables cognitivas, los siguientes aspectos de la imagen turística de Granada: "la ciudad ofrece muchas oportunidades para la aventura y el deporte", "sus costumbres y tradiciones son dignas de conocer", "es un lugar seguro para visitar", "dispone de numerosas atracciones culturales para visitar", "dispone de numerosas 
facilidades para ir de compras", "es un lugar con buena atención al turista y que cuida la calidad del servicio". Además los individuos que conforman este conglomerado no disponen de amplia información sobre la ciudad antes de efectuar el viaje.

Por otra parte, el conglomerado en el que predominan estudiantes americanos percibe una imagen más positiva de la ciudad de Granada que los miembros del otro conglomerado en relación a las siguientes variables: "Granada es un destino con gran riqueza histórica y patrimonial", "cuenta con una amplia oferta de restauración: bares, cafeterías, restaurantes, etc.", "presenta actividades culturales de interés"; "dispone de numerosas atracciones culturales para visitar"; "ofrece una oferta de alojamiento de calidad"; "Granada es una ciudad idónea para aprender español"; "es un destino con alta estabilidad política y social"; "existe una buena relación calidad/precio", "es un destino sin contaminación ambiental, limpio y cuidado" y "su clima resulta agradable".

Respecto al componente afectivo de la imagen, las variables consideradas en el cuestionario se analizan desde un punto de vista descriptivo, obteniendo las medias y desviaciones típicas. En definitiva, la imagen que perciben los estudiantes de español se asocia con una imagen del destino principalmente agradable $(\mathrm{M}=4,50$ $\mathrm{DT}=, 802)$, divertido $(\mathrm{M}=4,36 \mathrm{DT}=, 697)$, excitante $(\mathrm{M}=4,28 \mathrm{DT}=, 777) \mathrm{y}$ relajante $(\mathrm{M}=4,17 \mathrm{DT}=, 847)$.

La imagen global de Granada es muy positiva, valorándose con una puntuación de 4,18 sobre una escala Likert de 5 posiciones donde 1 es muy insatisfactorio y 5 muy satisfactorio.

\section{Pregunta abierta}

Para las respuestas obtenidas en la pregunta abierta se lleva a cabo un análisis de frecuencias en un documento de excel. Las palabras más mencionadas son "tapas" (13,49\% del total de encuestados), "ciudad bonita" (13,17\%) y "Alhambra" (7,31\%), seguidas de "diversión" (6,34\%), "ciudad cultural" $(5,20 \%)$, "relajación" (3,73\%), "ciudad histórica" (3,57\%), "sol" (2,60\%), "agradable" (2,43\%), "Sierra Nevada" (2,11\%), etc.

Posteriormente se realizan 11 bloques de agrupaciones de palabras que incluyen sustantivos y adjetivos con una connotación similar. Entre los tres bloques mejor valorados se encuentran "valoraciones positivas" que incluye aquellos adjetivos que describen la ciudad desde una perspectiva positiva, como ejemplo, los encuestados resaltan que Granada es una ciudad bonita, limpia, llena de vida, segura, etc. El segundo bloque mejor valorado se denomina "gastronomía" e incluye palabras como tapas, bar y cerveza. El tercer bloque alude a aquellos "lugares únicos" de Granada tales como la emblemática Alhambra, el famoso barrio del Albaicín y Sierra Nevada. 


\section{DISCUSIÓN}

Durante las últimas décadas, el estudio de la imagen turística ha sido uno de los temas más tratados en materia de Investigación Turística. Numerosos son los estudios que se han centrado en conocer la relación existente entre imagen-destino e intención de visita de un determinado lugar; diferencias entre la imagen de los visitantes antes y después del viaje o entre turistas que visitan por primera vez un lugar y personas que repiten la experiencia.

Tabla 4. Perfiles de los conglomerados. Test de ajuste estadístico chi-cuadrado

\begin{tabular}{|c|c|c|c|c|c|c|c|}
\hline & & \multicolumn{2}{|c|}{ Cluster 1} & \multicolumn{2}{|c|}{ Cluster 2} & \multicolumn{2}{|c|}{ Total } \\
\hline & & $\mathrm{N}$ & $\%$ & $\mathrm{~N}$ & $\%$ & $\mathrm{~N}$ & $\%$ \\
\hline \multirow{3}{*}{ 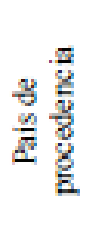 } & Americanos & 28 & $26,2 \%$ & 79 & $73,8 \%$ & 107 & $100 \%$ \\
\hline & Europeos & 43 & $87,8 \%$ & 6 & $12,2 \%$ & 49 & $100 \%$ \\
\hline & Asiáticos & 10 & $76,9 \%$ & 3 & $23,1 \%$ & 13 & $100 \%$ \\
\hline \multirow{3}{*}{$\begin{array}{l}\overline{8} \\
\bar{z} \\
\bar{z} \\
\frac{8}{8} \\
\bar{y} \\
\stackrel{z}{z}\end{array}$} & Básico & 23 & $82,1 \%$ & 5 & $17,9 \%$ & 28 & $100 \%$ \\
\hline & Intermedio & 42 & $\mathbf{5} 0,6 \%$ & 41 & $49,4 \%$ & 83 & $100 \%$ \\
\hline & Avanzado & 16 & $27,6 \%$ & 42 & $72,4 \%$ & 58 & $100 \%$ \\
\hline \multirow{6}{*}{ 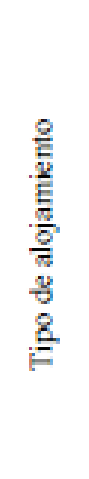 } & Residencia de estudiantes & 3 & $23,1 \%$ & 10 & $76,9 \%$ & 13 & $100 \%$ \\
\hline & Casa familia española & 20 & $25,6 \%$ & 58 & $74,4 \%$ & 78 & $100 \%$ \\
\hline & Piso de estudiantes & 44 & $81,5 \%$ & 10 & $18,5 \%$ & 54 & $100 \%$ \\
\hline & Establecimiento hotelero & 1 & $100 \%$ & 0 & $0 \%$ & 1 & $100 \%$ \\
\hline & Apartamento de alquiler & 13 & $59,1 \%$ & 9 & $40,9 \%$ & 22 & $100 \%$ \\
\hline & Otro tipo & 0 & $0 \%$ & 1 & $100 \%$ & 1 & $100 \%$ \\
\hline
\end{tabular}

Fuente: Elaboración propia 
Tabla 5. Análisis descriptivo de las variables afectivas

Fuente: Elaboración propia

\begin{tabular}{l|c|c} 
Variables afectivas & $\mathrm{M}$ & $\mathrm{DT}$ \\
\hline Aburrido - Divertido & 4,36 &, 697 \\
Estresante - Relajante & 4,17 &, 847 \\
Deprimente - Excitante & 4,28 &, 777 \\
Desagradable - Agradable & 4,50 &, 802
\end{tabular}

Tabla 6. Análisis descriptivo de la imagen

Fuente: Elaboración propia

\begin{tabular}{l|c|c|} 
& $\mathrm{M}$ & $\mathrm{DT}$ \\
\hline Imagen global & 4,18 &, 741
\end{tabular}

Tabla 7. Frecuencia en número relativos de las palabras referenciadas en la pregunta abierta

\begin{tabular}{l|l} 
Palabras $(\mathrm{N}=615)$ & $\%$ \\
\hline Tapas & $13,49 \%$ \\
Ciudad bonita & $13,17 \%$ \\
Alhambra & $7,31 \%$ \\
Diversión & $6,34 \%$ \\
Ciudad cultural & $5,20 \%$ \\
Relajación & $3,73 \%$ \\
Ciudad histórica & $3,57 \%$ \\
Sol & $2,60 \%$ \\
Agradable & $2,43 \%$ \\
Sierra Nevada & $2,11 \%$
\end{tabular}

Fuente: Elaboración propia 
Tabla 8. Agrupaciones de las palabras descriptivas

\begin{tabular}{|l|l|c|c|}
\hline \multicolumn{1}{|c|}{ Agrupaciones } & \multicolumn{1}{|c|}{ Palabras descriptivas } & $\mathrm{N}$ & \multicolumn{1}{c|}{$\%$} \\
\hline Valoraciones positivas & $\begin{array}{l}\text { Bonita, barata, limpia, interesante, llena de vida, alegre, } \\
\text { agradable, acogedora, única, pintoresca, auténtica, segura, } \\
\text { inolvidable, etc. }\end{array}$ & 197 & $32,03 \%$ \\
\hline Gastronomía & Tapas, bar, cerveza, pescado, jamón & 89 & $14,47 \%$ \\
\hline Lugares únicos & Albaicín, Alhambra, Sierra Nevada & 67 & $10,89 \%$ \\
\hline $\begin{array}{l}\text { Referencias } \\
\text { culturales/históricas }\end{array}$ & $\begin{array}{l}\text { Ciudad cultural, ciudad histórica, ciudad árabe, ciudad } \\
\text { universitaria, árabe, Isabel y Fernando }\end{array}$ & 65 & $10,56 \%$ \\
\hline Ocio & Vida nocturna, música, diversión, centros comerciales & 57 & $9,27 \%$ \\
\hline Sensaciones & $\begin{array}{l}\text { Emoción, fascinación, felicidad, inspiración, placer, } \\
\text { relajación }\end{array}$ & 42 & $6,83 \%$ \\
\hline Estereotipos & $\begin{array}{l}\text { Flamenco, toros, siesta, típica de Andalucía, vacaciones, } \\
\text { España }\end{array}$ & 32 & $5,20 \%$ \\
\hline Clima & Sol, clima cálido & 28 & $4,55 \%$ \\
\hline Población & Agradable, hospitalaria, acogedor, joven, multicultural & 25 & $4,07 \%$ \\
\hline Referencias al deporte & Aventura, deporte, fútbol & 7 & $1,14 \%$ \\
\hline Valoraciones negativas & Abarrotada de gente, sucia, estresante & 6 & $0,98 \%$ \\
\hline Total & & 615 & $100 \%$ \\
\hline
\end{tabular}

Fuente: Elaboración propia

En cambio, escasos son los trabajos que tratan sobre el impacto que la creciente industria de programas de estudios en el extranjero tiene en la percepción de la imagen de un país o determinada ciudad (Chen y Kerstetter, 1999; Gertner, 2010). En este sentido, esta investigación permite indagar sobre este ámbito conociendo la imagen que un grupo de estudiantes de español percibe sobre la ciudad de Granada. El hecho de considerar como muestra de la investigación a este segmento de la población presenta una novedad respecto a los trabajos previos, en los que generalmente no se considera a un único segmento de la población como objeto de estudio.

En primer lugar, los resultados del presente trabajo señalan la existencia de diferencias en la imagen percibida de la ciudad de Granada en función de la nacionalidad de los estudiantes. En este sentido, los resultados obtenidos indican que los americanos perciben de manera más positiva que los europeos y asiáticos la calidad del alojamiento de la ciudad, las facilidades para ir de compras, las costumbres y tradiciones, las actividades de aventura y deporte que se pueden practicar, así como la estabilidad política y social del destino. Los americanos también señalan la idoneidad de la ciudad para aprender español. Este último hecho se ve respaldado por el trabajo de Criado (2003) en el que se pone de manifiesto el creciente interés que la lengua española suscita en Estados Unidos. 
Figura 3. Distribución de los clúster
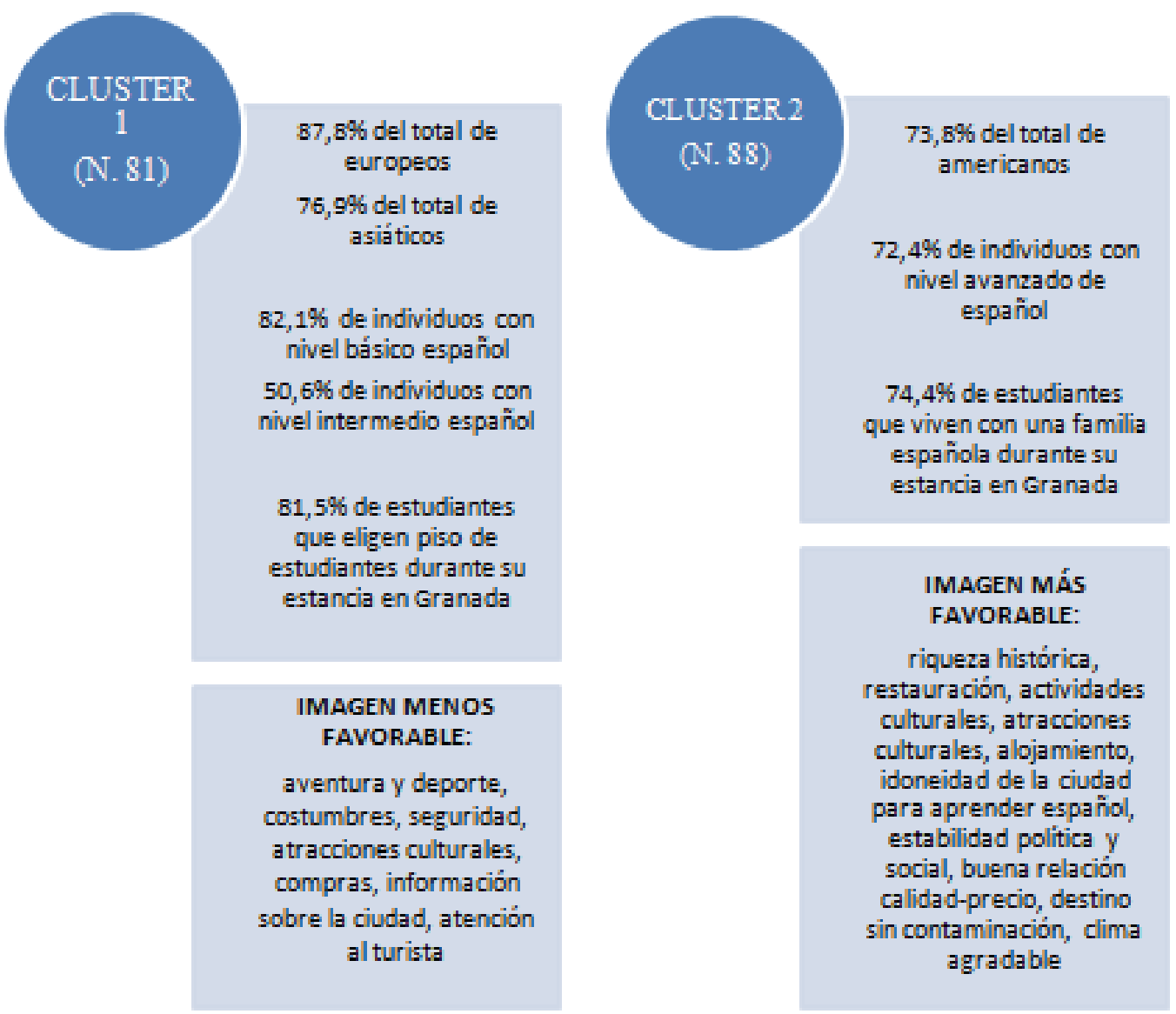
que eligen piso de estudiantes durante su estancia en Granada

IMAGEN MÁS FAVORABLE:

riqueza histórica, restauración, actividades culturales, atracciones culturales, alojamiento, idoneidad de la ciudad para aprender español, estabilidad politica y social, buena relación calidad-precio, destino sin contaminación, clima agradable

Fuente: Elaboración propia

En cambio, los estudiantes europeos son los que menos asocian los espectáculos taurinos como un rasgo característico de la ciudad de Granada. Esto puede deberse a la cercanía cultural y la mayor familiaridad con el destino, lo cual reafirma la teoría de Fayeke y Crompton (1991), quienes ponen de relieve que cuanto mayor es la familiaridad del individuo con el destino, más precisas serán las percepciones del mismo, lo que implica una reducción de los estereotipos de la imagen. En este caso, las escasas relaciones históricas entre Andalucía y países asiáticos conlleva que el destino resulte poco familiar para el turista asiático y que por tanto se haga más patente la existencia de estereotipos (Gómez, 2012).

Respecto a la seguridad, los estudiantes asiáticos perciben la ciudad de manera menos segura que los estudiantes del resto de nacionalidades. Este hecho se constata también en los estudios de Gómez (2012) y Jimeno (2005). Esto puede 
explicarse por el hecho de que estos turistas proceden de ciudades muy seguras y no perciben el riesgo de hurtos existentes en la ciudad, lo que provoca que estos turistas sean un objetivo fácil por parte de los delincuentes (Gómez, 2012).

Gráfico 1. Conglomerado 1. T-student

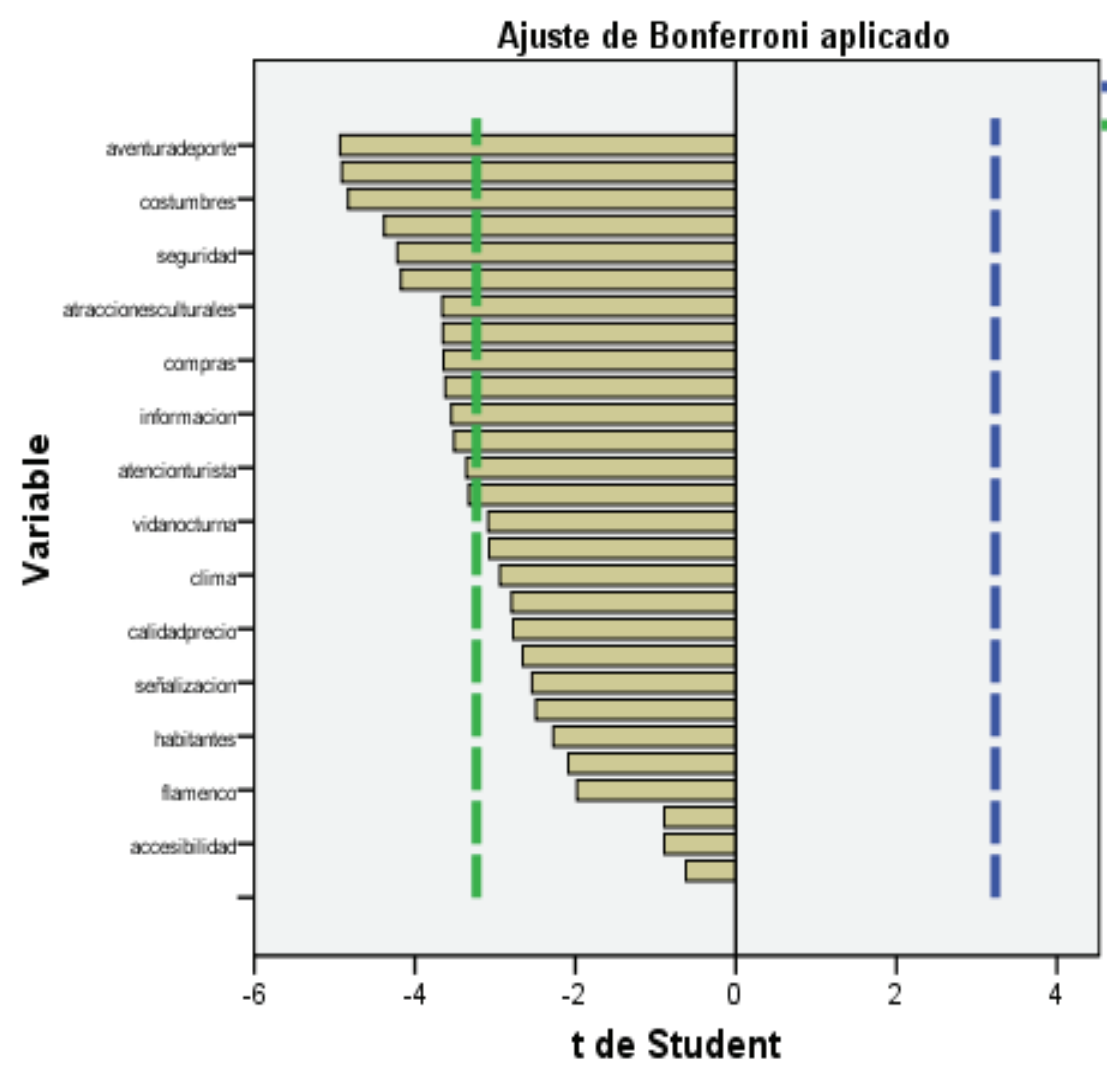

Fuente: Elaboración propia

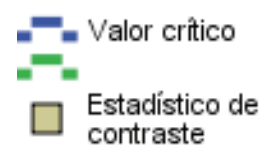

Por otra parte, los estudiantes procedentes de Asia identifican los espectáculos taurinos como uno de los rasgos más característicos de la ciudad frente a los americanos y europeos. Este hecho puede explicarse por la imagen estereotipada que los turistas asiáticos poseen tanto de España como de Andalucía, y que coincide con los resultados del estudio llevado a cabo por Gómez (2012). Esta investigadora analiza diversas publicaciones japonesas (folletos turísticos, guía de viajes de España y un libro de conversación en español) relacionadas con viajes a España. En todos ellos se resalta una imagen de Andalucía vinculada con 
determinados estereotipos, como el ambiente festivo, la influencia islámica en los monumentos, el flamenco, los toros, y la gastronomía basada en las tapas y el jamón serrano. Asimismo, el trabajo de investigación de Carrascosa (2003) sobre la imagen de España en Japón, destaca la cultura taurina como uno de los elementos claves de la imagen percibida de este país por este colectivo. Igualmente, Lamo de Espinosa (1996) indica que los toros son uno de los tópicos que asocian los extranjeros a la imagen de España.

Gráfico 2. Conglomerado 2. T-student

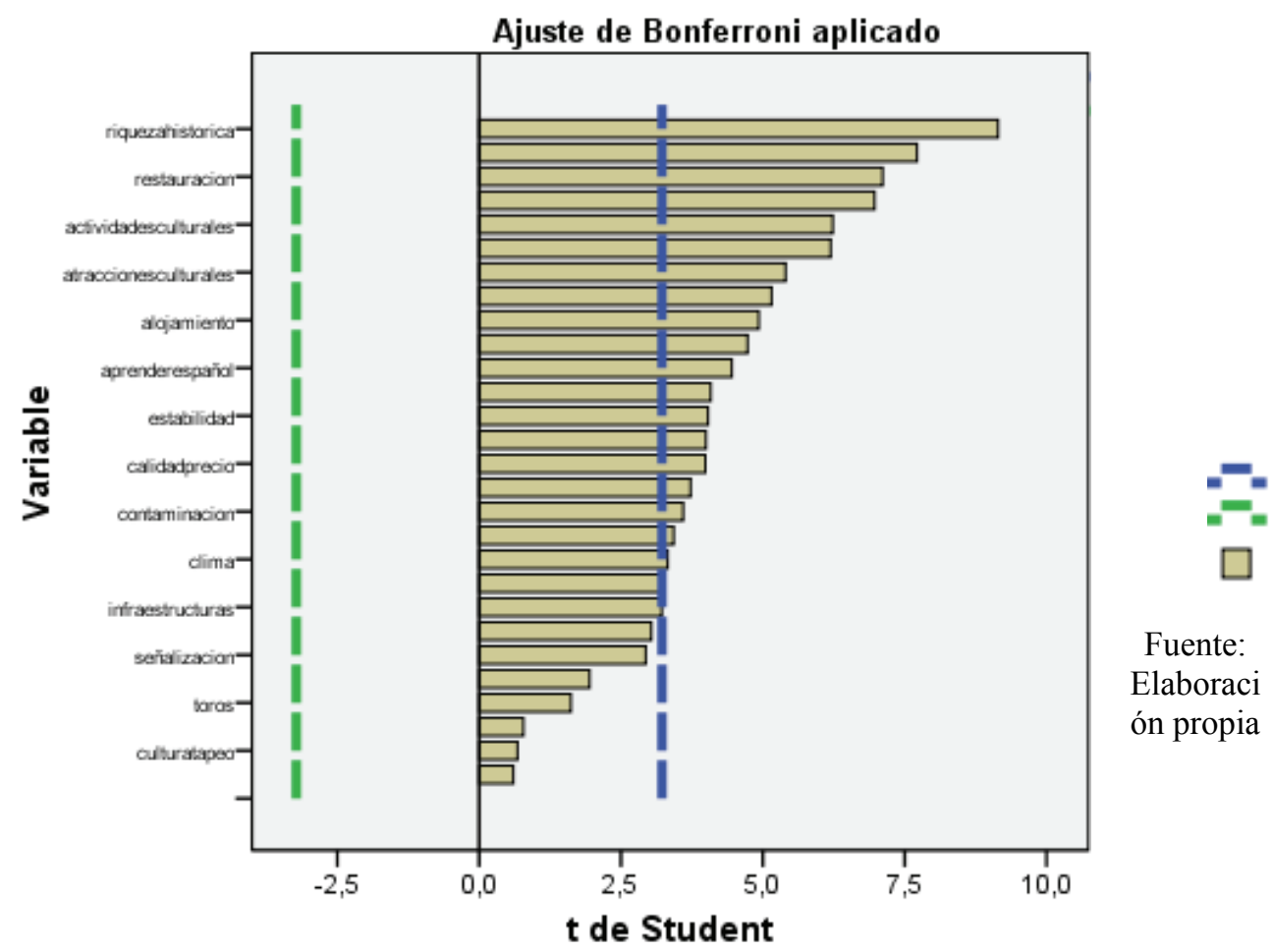

En segundo lugar, la presente investigación trata de determinar la posible existencia de agrupaciones o "clúster" de estudiantes que compartan una serie de particularidades y unas percepciones similares de la imagen del destino, directamente relacionadas con las características de cada clúster "Ver figura 3". La utilización de esta técnica de análisis estadístico constituye una de las principales aportaciones a las investigaciones previas, en las que predominan análisis de carácter más descriptivo. Generalmente, los investigadores establecen un perfil sociodemográfico de la muestra. A continuación, realizan un análisis factorial exploratorio de los componentes principales con rotación varimax para reducir las 
dimensiones e identificar los factores determinantes en la formación de la imagen de un destino. Finalmente, se lleva a cabo un análisis anova para determinar la existencia o no de diferencias significativas de los componentes que integran la imagen respecto a diversas variables sociodemográficas como el género, la edad, el país de procedencia, etc. Otros estudios emplean análisis de regresión para comprobar hasta qué punto los factores identificados en el análisis factorial explican la imagen de un destino (Ahmed, 1991; Andrade, 2011; Baloglu, 1997; Baloglu y McCleary, 1999a, 1999b; Beerli y Martín, 2004a, 2004b, 2004c; Bigné y Sánchez, 2001; Bigné, Sánchez y Sanz, 2009; San Martín et al., 2006; San Martín y Rodríguez 2008, 2010a, 2010b; Mackay y Fesenmaier, 1997; Moreno et al., 2012; Sahin y Baloglu, 2011; Sirakaya,Sönmez. y Choi, 2001; Sönmez y Sirakaya, 2002; Suárez, 2012).

En cambio, son mínimos los trabajos en los que se engloba a la muestra en agrupaciones o clúster que se caracterizan por incluir a individuos que comparten un perfil psicosocial similar y una imagen semejante de un determinado lugar (Agapito, Mendes y Valle, 2010; Andreu, Bigné y Cooper, 2000; Castro, Martín y Martín, 2007; Goodrich, 1978; San Martín y Rodríguez, 2008, 2010a, 2010b). Los resultados obtenidos en el presente trabajo en relación con los clúster difieren de los estudios previos. Esto puede explicarse por el hecho de que la muestra considerada en este trabajo no ha sido considerada en investigaciones previas y que al tratarse de un grupo de estudiantes cuya motivación principal es aprender español, muestra unas características muy específicas.

Este trabajo de investigación presenta varias limitaciones. En primer lugar, el carácter exploratorio del análisis clasificatorio estadístico en conglomerados de estudiantes o clúster. Hubiera sido recomendable aumentar el tamaño de la muestra lo que hubiera posibilitado confirmar la distribución de los clúster y generalizar los resultados del presente trabajo. En segundo lugar, el uso de una muestra de estudiantes no permite la generalización de los resultados más allá de la población de estudiantes. Además, la utilización de este tipo de muestra conlleva que los individuos tengan una edad, una ocupación y un nivel de formación similares. En tercer lugar, en este estudio se ha considerado tan sólo a los turistas actuales sin incluir a los potenciales. Como consecuencia, la imagen estudiada hace referencia a la formada tras la experiencia vacacional.

A raíz de los resultados obtenidos, se plantean futuras líneas de investigación. (i) La primera de ellas hace referencia al carácter tranversal de la presente investigación, lo que conlleva que los resultados obtenidos son sólo representativos para un período específico de tiempo. Por tanto, es necesario llevar a cabo un estudio longitudinal en el que se considere una muestra de estudiantes más amplia y representativa de la población objeto de estudio. (ii) La realización del estudio a estudiantes potenciales, es decir, a aquellos que aún no han visitado la ciudad de Granada. Esto se explica por el hecho de que encuestar a estudiantes que ya han decidido visitar el destino puede condicionar los resultados obtenidos. (iii) La 
aplicación del estudio a otras ciudades nacionales consideradas como destinos de turismo idiomático, lo que permitiría generalizar los resultados a nivel nacional.

En definitiva, hoy en día resulta de vital importancia que un destino desarrolle una imagen favorable, fuerte y única que permita crear valor en la mente del turista y le permita posicionarse en el actual mercado competitivo. Asimismo, la importancia turística de la lengua española contribuye notablemente a proyectar y reforzar internacionalmente la imagen de un destino.

\section{BIBLIOGRAFÍA}

AGAPITO, D., MENDES, J. y VALLE, P. (2010). Destination image. Perspectives of tourists versus residents. European Journal of Tourism, Hospitality and Recreation, Vol. 1, no 1.p. 90-109

AHMED, Z.U. (1991). The influence of the components of a state's tourist image on product positioning strategy. Tourism Management,Vol.12, p. 331-340.

ANDRADE, M.J. (2011). La formación de la imagen turística desde la perspectiva de la distancia cultural del viajero. Estudios y Perspectivas en Turismo, Vol. 20, p. $1237-1257$.

ANDREU, L., BIGNÉ, J.E. y COOPER, C. (2000). Projected and perceived image of Spain as a tourist destination for British travelers. Journal of Travel \&Tourism Marketing, Vol. 9, no 4. p. 47-67.

BALOGLU, S. (1997). The relationship between destination images and sociodemographic and trip characteristics of international travellers. Journal of Vacation Marketing, Vol. 3, no 3. p. 221-233.

BALOGLU0, S. y McCLEARY, K.W. (1999a). "A model of destination formation". Annals of Tourism Research, Vol. 26, no 4.p. 868-897.

BALOGLU, S. y McCLEARY, K.W. (1999b). "U.S International pleasure travelers' images of four Mediterranean destinations: a comparison of visitors and nonvisitors". Journal of Travel Research, Vol. 38, no 2.p.144-152.

BEERLI, A. y MARTÍN, J.D. (2004a). "Cómo influyen las fuentes de información en la imagen percibida de los destinos turísticos". Revista Española de Investigación de Marketing, Vol. 8, no 2. p. 7-34.

BEERLI, A. y MARTÍN, J.D. (2004b). Factors influencing destination image. Annals of Tourism Research, Vol. 31, no 3. p. 657-681.

BEERLI, A. y MARTÍN, J.D. (2004c). Tourists' characteristics and the perceived image of tourist destinations: a quantitative analysis - a case study of Lanzarote, Spain. TourismManagement, Vol. 25, no 5, p. 623-636.

BIGNÉ, E. y SÁNCHEZ, I. (2001). Evaluación de la imagen de destinos turísticos: una aplicación metodológica en la Comunidad Valenciana. Revista Europea de Dirección y Economía de la Empresa, Vol. 10, no 3, p. 189-200. 
BIGNÉ, E., SÁNCHEZ, I. y SÁNCHEZ, J. (2001). Tourism image, evaluation variables and after purchase behaviour: inter-relationship. Tourism Management, Vol. 22, p. 607-616.

BIGNÉ, E., SÁNCHEZ, I. y SANZ. S. (2009). The functional-psychological continuum in the cognitive image of a destination: a confirmatory analysis. Tourism Management, Vol. 30, p. 715-723.

CARRASCOSA, A. (2003). La imagen de España en Japón. Boletín Económico de ICE (2770)

CASTAÑO, J.M., MORENO, A. y CREGO, A. (2006). Factores psicosociales y formación de imágenes en el turismo urbano: un estudio de caso sobre Madrid. PASOS Revista de Turismo y Patrimonio Cultural, Vol. 4, no 3. p. 287-299.

CASTRO, C., MARTÍN, E. y MARTÍN, D. (2007). The influence of market heterogeneity on the relationship between a destination's image and tourists' future behavior. Tourism Management, Vol. 28, p. 175-187.

CHEN, P. y KERSTETTER, D.L. (1999) 'International students' image of rural Pennsylvania as a travel destination". Journal of Travel Research, Vol. 37, no 3. p. 256-266.

CRIADO, M.J. (2003). La lengua española en Estados Unidos: luces y sombras. Boletín del Real Instituto Elcano de Estudios Internacionales y Estratégicos, Vol. 17, p. 1-22.

CROMPTON, J.L. (1979). "An assessment of the image of Mexico as a vacation Destination and the influence of geographical location upon the image". Journal of Travel Research, Vol. 17, p. 18-23.

DEL BARRIO, S., LUQUE, T. y RODRÍGUEZ, M.A. (2009). La modelización de la imagen de la ciudad desde la perspectiva de los líderes de opinión externos. Revista Eure, Vol. 24, no 106. p. 9-28.

ECHTNER, C.M. y RITCHIE, J.R.B. (2003). "The meaning and measurement of destination image". The Journal of Tourism Studies, Vol 14, no 1.p. 37-48.

FAYEKE, P.C. y CROMPTON, J.L. (1991). "Image differences between prospective, first-time, and repeat visitors to the Lower Rio Grande Valley". Journal of Travel Research, Vol. 30, no 2.p. 10-16.

GALLARZA, M.G., GIL, I. y CALDERÓN, H. (2002). "Destination image: towards a conceptual framework". Annals of Tourism Research, Vol. 29, no 1. p. 56-78.

GARTNER, W.C. (1993). "Image formation process". Journal of Travel \& Tourism Marketing, Vol 2, no 2-3. p. 191-215.

GERTNER, R. (2010). "Similarities and differences of the effect of country images on tourist and study destinations". Journal of Travel Research \& Tourism Marketing, Vol. 27, p. 383-395.

GOODRICH, J.N. (1978). The relationship between preferences for and perceptions of vacation destinations: application of a choice model. Journal of Travel Research, Vol. 17, no 2. p. 8-13. 
GÓMEZ, A. (2010). "La imagen de Andalucía en el turismo cultural: el caso de Japón". En III Jornadas de Investigación en Turismo "Nuevas perspectivas del turismo para la nueva década".p. 643-663. Sevilla: Universidad de Sevilla. Escuela Universitaria de Estudios Empresariales

GUNN, C. (1988): Vacationscape. Designing Tourist Regions. 2nd Ed. New York: V.N. Reinhold.

HERNÁNDEZ, J.M. y CAMPÓN, A.M. (2010). "Evolución del turismo idiomático en España. Un sector estratégico en auge". Revista de Estudios Turísticos, Vol. 186, p .45-68.

JIMENO, M. (2005). El turismo cultural en la gestión de la Marca España. Real Instituto Elcano de Estudios Internacionales y Estratégicos. Documento de Trabajo, 39, 1-41. Disponible en:

http://www.realinstitutoelcano.org/documentos/214/214_JimenoVinyesPDF.pdf

KIM, S.S., y LEE, C.L. (2002). Push and pull relationship. Annals of Tourism Research, Vol. 29, no 1. p. 257-260.

LAMO DE ESPINOSA, E. (1996). La Imagen de España en el Exterior. Conclusiones de una investigación. Dialnet. Universidad de La Rioja. Disponible en: http://dialnet.unirioja.es/servlet/articulo?codigo $=201170$

LEE, G. y LEE, C.K. (2009). Cross-cultural comparison of the image of Guam perceived by Korean and Japanese leisure travelers: Importance - performance analysis. Tourism Management, Vol. 30, p. 922-931.

MACKAY, K.J. y FESENMAIER, D.R. (1997). Pictorial Element of Destination in Image Formation. Annals of Tourism Research, Vol. 24, no 3. p. 537-565.

MORENO, S., BEERLI, A. y LEDESMA, J. (2012). "Entender la imagen de un destino turístico: factores que la integran y la influencia de las motivaciones". Criterio Libre, Vol. 10, no 16. p. 115-142.

MORENO, S., BEERLI, A. y MARTÍN J.D. (2004). La imagen de Gran Canaria como destino turístico. Vector Plus, Vol. 31, p. 71-78.

ORGANIZACIÓN MUNDIAL DEL TURISMO(1998). Introducción al Turismo. Madrid, España: OMT.

PARDO ABAD, C. (2011). "El turismo idiomático en España como potenciador cultural en ciudades patrimoniales: El caso de Salamanca y Alcalá de Henares". Cuadernos de Turismo, Vol. 27, p. 701-723.

PIKE, S. (2002). Destination image analysis: a review of 142 papers from 19732000. Tourism Management, Vol. 23, no 5. p. 541-549.

RIAL, A., GARCÍA, A. y VARELA, J. (2008). Una aplicación metodológica para el estudio de la imagen de marca de un destino turístico. PASOS Revista de Turismo y Patrimonio Cultural, Vol. 6, no 1.p. 1-10

SAHIN, S. y BALOGLU S. (2011). Brand personality and destination image of Istanbul. Anatolia, Vol. 22, no 1. p. 69-88. 
SAN MARTÍN, H. y RODRÍGUEZ, I. (2008). Exploring the cognitive-affective nature of destination image and the role of psychological factors in its formation. Tourism Management, Vol. 29, p. 263-277.

SAN MARTÍN, H. y RODRÍGUEZ, I. (2010a). Los factores estímulo y personales como determinantes de la formación de la imagen de marca de los destinos turísticos: un estudio aplicado a los turistas que visitan un destino vacacional. Cuadernos de Economía y Dirección de la Empresa, Vol. 43, p. 37-63.

SAN MARTÍN, H. y RODRÍGUEZ, I. (2010b). Un enfoque de gestión de la imagen de marca de los destinos turísticos basado en las características del turista. Revista de Análisis Turístico, Vol. 9, p. 5-13.

SAN MARTÍN, H., RODRÍGUEZ, I. y VÁZQUEZ, R. (2006). "Análisis de la imagen en turismo mediante técnicas estructuradas y no estructuradas: implicaciones competitivas para los destinos turísticos". Revista Asturiana de Economía, Vol. 35, p. 69-90.

SIRAKAYA, E., SÖNMEZ, S. y CHOI, H.S. (2001). Do destination image really matter? Predicting destination choices of student travelers. Journal of Vacation Marketing, Vol. 7, no 2. p. 125-142.

SÖNMEZ, S. y SIIRAKAYA, E. (2002). A distorted destination image? The case of Turkey. Journal of Travel Research, Vol. 41, no 2. p. 185-196.

SUÁREZ, M.J. (2012). El efecto moderador de la procedencia cultural del turista en la percepción de la imagen del destino. Sociología.Revista da Facultade de Letras da Universidade do Porto, Vol.23,p. 11-35.

WANG, C.Y. y HSU, M. (2010). The relationships of destination image, satisfaction, and behavioral intentions: an integrated model. Journal of Travel \& Tourism Management, Vol. 27, p. 829-843.

YOON, Y., y UYSAL, M. (2005). An examination of the effects of motivation and satisfaction on destination loyalty: a structural model. Tourism Management, Vol. 26, p. 45-56. 